\title{
In vitro efficacy of Metarhizium anisopliae sensu lato against unfed Amblyomma parvum (Acari: Ixodidae)
}

\author{
Marcos Valerio Garcia ${ }^{1,2}$ - Vinicius da Silva Rodrigues ${ }^{3,4}$. Antônio Carlos Monteiro ${ }^{5}$. \\ Lucas Detogni Simi ${ }^{5}$. Leandro de Oliveira Souza Higa ${ }^{2,6}$. Maria Marlene Martins ${ }^{4}$. \\ Nancy Prette ${ }^{7}$. Dinalva Alves Mochi ${ }^{5} \cdot$ Renato Andreotti $^{8} \cdot$ Matias Pablo Juan Szabó $^{4}$
}

Received: 20 July 2018 / Accepted: 20 October 2018 / Published online: 12 November 2018

(c) Springer Nature Switzerland AG 2018

\begin{abstract}
Amblyomma parvum Aragão (Acari: Ixodidae) is a tick species found with wide distribution in the Neotropical region. Even though it is a wildlife-related tick, it is also a frequent parasite of domestic animals, is aggressive to human beings and may harbor pathogenic microorganisms. Therefore, it is a target species for control on domestic animals, particularly those at the rural-wildlife interface. Herein, the efficacy of two isolates (E9 and IBCB 425) of an entomopathogenic fungus, Metarhizium anisopliae sensu lato, already evaluated for ticks that parasitize domestic animals, was tested against unfed A. parvum adults. Both isolates displayed high acaricidal efficacy after immersion in fungal conidial suspensions for 5 min. Isolate E9 killed all ticks by the 7th day post-treatment, and isolate IBCB 425 did so by the 11 th day. Tick mortality of 80 and $90 \%$ was achieved as early as the 3rd and 4th days, respectively, with both treatments. Thus, if a commercial M. anisopliae s.l. acaricide against domestic animal ticks is developed, it would also be effective against $A$. parvum.
\end{abstract}

Marcos Valerio Garcia

marcosvagar@gmail.com

1 Bolsista DCR, Fundect-Governo do Estado de Mato Grosso do Sul, Campo Grande, Brazil

2 Laboratório de Biologia do Carrapato, Embrapa Gado de Corte, Campo Grande, Mato Grosso Do Sul, Brazil

3 Programa de Pós-Graduação em Imunologia e Parasitologia Aplicadas, Universidade Federal de Uberlândia, Uberlândia, Minas Gerais, Brazil

4 Laboratório de Ixodologia, Faculdade de Medicina Veterinária, Universidade Federal de Uberlândia, Av. Pará, 1720/Campus Umuarama-Bloco 2T, Uberlândia, Minas Gerais CEP 38400-902, Brazil

5 Laboratório de Microbiologia Agropecuária, Departamento de Produção Vegetal Faculdade de Ciências Agrárias e Veterinárias de Jaboticabal, Via de Acesso Prof. Paulo Donato Castellane s/n, Jaboticabal, São Paulo CEP 14884-900, Brazil

6 Programa de Pós-Graduação em Doenças Infecciosas e Parasitárias, Universidade Federal de Mato Grosso do Sul, Campo Grande, Mato Grosso Do Sul, Brazil

7 Universidade Federal da Paraíba, Campus II, Ciências Agrárias, Areia, Paraíba, Brazil

8 Empresa Brasileira de Pesquisa Agropecuária, Embrapa Gado de Corte, Campo Grande, Mato Grosso do Sul, Brazil 
Keywords Amblyomma parvum $\cdot$ Acaricide $\cdot$ Entomopathogenic fungus $\cdot$ Control

\section{Introduction}

Amblyomma parvum Aragão (Acari: Ixodidae) is a Neotropical tick species found in a range from Argentina to Mexico, and three distinct lineages can be distinguished within its broad geographic distribution (Lado et al. 2016). Within its range, it is associated with dry and hot environments where adults and immature stages parasitize medium- and largesized mammals and rodents, respectively (Nava et al. 2008). Importantly, A. parvum is frequently found, together with livestock ticks, on several domestic animals, including cattle, horses, dogs and goats (Szabó et al. 2007; Nava et al. 2008; Ramos et al. 2016).

Furthermore, under experimental settings, several domestic and laboratory animals can sustain their entire parasitic life cycle alone (Olegário et al. 2011). In fact, parasitism of domestic animals by A. parvum may be enhanced by the mandatory conservation of natural resources within rural properties throughout Brazil and the increased intermingling of domestic and wild animals. Importantly, A. parvum ticks, particularly adults, were shown to be aggressive to humans (Ramos et al. 2014) as well as potential vectors of several pathogens. In this regard, this tick species has been found to be infected with Coxiella burnetii, Ehrlichia chaffeensis and Candidatus Rickettsia andeanae (Nieri-Bastos et al. 2014; Pacheco et al. 2013; Tomassone et al. 2008).

A relevant feature of $A$. parvum is that it is a small brown tick lacking ornamentation, and adults are, at first sight, easily confused with similarly small brown ticks such as Rhipicephalus sanguineus or with nymphs of other Amblyomma species. Therefore, it is prone to go unnoticed or confused with other tick species, and thus, domestic animal and human infestations are probably underreported.

Tick control on domestic animals relies heavily on acaricides, but with drawbacks. The resistance of tick populations to several acaricidal compounds is a common occurrence after massive use (Higa et al. 2015), and environmental toxicity, with effects on nontarget organisms, is of great concern (De Meneghi et al. 2016). This last aspect is especially important if one considers the abovementioned proximity of wildlife and domestic animals and mixed infestations with domestic and wildlife ticks. Among more environmentally friendly alternative tick control measures, entomopathogenic fungi such as Metarhizium anisopliae sensu lato were shown to be effective against ticks in laboratory trials. However, these trials were overwhelmingly conducted with ticks from domestic animals such as Rhipicephalus microplus and R. sanguineus (Bittencourt et al. 1999; Garcia et al. 2005, 2011). Herein we tested the acaricidal efficacy of $M$. anisopliae s.l. on unfed adult $A$. parvum ticks to evaluate whether a wildlife tick with a great potential to parasitize domestic animals exhibits similar sensitivity to this fungus under laboratory conditions as shown for other tick species.

\section{Materials and methods}

\section{Metarhizium anisopliae s.I. fungus}

Isolates E9 and IBCB 425 of $M$. anisopliae s.l. fungus, previously shown to have pathogenic effects in vitro against ticks from domestic animals (Monteiro et al. 1998; Garcia 
et al. 2004, 2005), were used. Isolates for the experiments were acquired from the stock culture of the Microbiology Laboratory of the Plant Production Department, Faculty of Agrarian and Veterinary Sciences (FCAV)-São Paulo State University (Unesp), Jaboticabal City, São Paulo State, Brazil. These isolates were cultivated in Petri dishes containing a potato-dextrose-agar medium (PDA) at $27^{\circ} \mathrm{C}$ in the absence of light for 12 days. Conidia from the surface of colonies were transferred to test tubes containing $20 \mathrm{~mL}$ of Tween $80^{\circledR}, 0.1 \%$ solution $(\mathrm{v} / \mathrm{v})$. The number of conidia per gram of rice in the suspensions was determined using a Neubauer chamber. The same suspensions described by Francisco et al. (2006) were used to determine conidial viability. Overall viability was shown to be $95 \%$.

\section{Amblyomma parvum ticks}

Unfed adult ticks were acquired from a laboratory colony of A. parvum ticks kept in the Ixodology Laboratory of the Veterinary Faculty of the Federal University of Uberlândia, Minas Gerais, Brazil. Ticks from this colony originated from Araguapaz, Goiás Brazil, and were maintained by feeding them on tick-bite naïve New Zealand rabbits as described previously (Olegário et al. 2011). Laboratory tick colony maintenance procedures were approved by the ethics committee for animal usage of the Federal University of Uberlândia, Uberlândia, Minas Gerais, Brazil (protocol number 97/11).

\section{Acaricide efficacy tests}

Overall, 120 adult ticks (60 males and 60 females) were divided into two experimental groups and one control group. Each treatment was performed four times each with groups of ten ticks (quadruple testing). Treatment consisted of the immersion of ticks for $5 \mathrm{~min}$ in the fungal conidial suspension (either $M$. anisopliae s.l. E9 or TBCB test groups) at a concentration of $1.0 \times 10^{9}$ conidia/ml or the conidia suspension vehicle alone (control group). After immersion, the ticks were paper dried and individually stored in plastic tubes at $25{ }^{\circ} \mathrm{C}$ and $80 \%$ relative humidity under a $12: 12 \mathrm{~h}$ light regimen. Tick infection and mortality were evaluated until the 11th day post-immersion. Tick infection was determined by visual inspection and mortality by the immobility of ticks stimulated by slight blowing.

\section{Statistical analysis}

The analysis of variance was conducted using the Kruskal-Wallis test. The level of significance was set at $5 \%$.

\section{Results}

Both M. anisopliae s.l. isolates induced $100 \%$ mortality in unfed adult A. parvum ticks. Isolate E9 killed all ticks by the 7th day post-treatment, and isolate IBCB 425 did so by the 11th day (Fig. 1). Nevertheless, the results did not differ significantly; both isolates induced rapid mortality, and $80 \%$ and $90 \%$ of tick mortality was achieved as soon as the 3rd and 4th days, respectively, with both treatments. Control ticks began to die on the 5 th day, and by day 11 after treatment, more than $70 \%$ remained alive. 


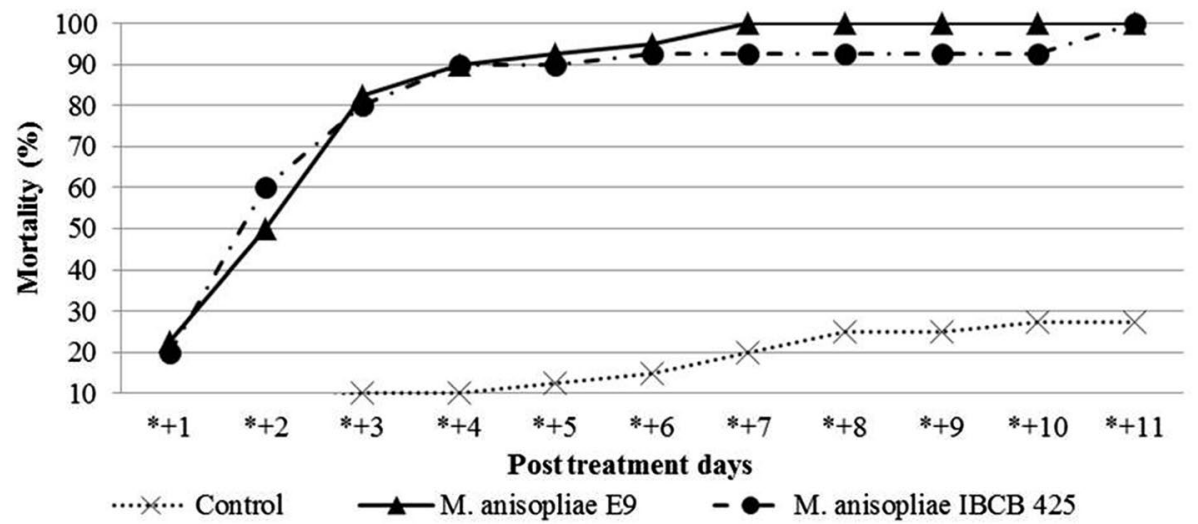

Fig. 1 Cumulative daily mortality rate of unfed adult Amblyomma parvum ticks following immersion in either Metarhizium anisopliae s.1. E9 or IBCB 425 isolate conidial solutions or control solution

The number of $A$. parvum ticks found dead each post-treatment day after immersion in either M. anisopliae s.l. isolate E9 or IBCB 425 conidial solutions or the control solution is displayed in Table 1.

\section{Discussion}

A high and fast mortality of unfed adult A. parvum ticks was observed after immersion in a conidial suspension of $M$. anisopliae s.l. isolates. This high mortality may have been caused by the high sensitivity of $A$. parvum to fungal infection and/or by the high concentration of the conidial suspension. Amblyomma parvum is a tick species found in xeric environments (Nava et al. 2008), and it can be speculated that it has not evolved appropriate defense mechanisms against parasites associated with moist surroundings, such as fungi. At the same time, the conidial suspension used was at a high concentration in relation to some other acaricidal tests (Basso et al. 2005; Polar et al. 2005; Lopes et al. 2007; Garcia et al. 2011) but not to all (Bittencourt et al. 2003). A conidial concentration increase was shown to be directly related to an increase in the tick mortality rate (Lopes et al. 2007). In this regard, conidial concentration in commercial products should ideally be high enough to attain the desired efficacy against several species if mixed tick infestations occur, as found in the case of A. parvum on domestic animals (Szabó et al. 2007; Ramos et al. 2016).

Table 1 Number of Amblyomma parvum ticks found dead each post treatment day after immersion in either Metarhizium anisopliae s.1. isolate E9 or IBCB 425 solutions or a control solution

\begin{tabular}{llrrrrrrrrrrr}
\hline Isolate & \multicolumn{9}{l}{ Number of ticks found dead each post treatment day } & & Total \\
\cline { 2 - 11 } & +1 & +2 & +3 & +4 & +5 & +6 & +7 & +8 & +9 & +10 & +11 \\
\hline E9 & 9 & 11 & 13 & 3 & 1 & 1 & 2 & 0 & 0 & 0 & 0 & 40 \\
IBCB 425 & 8 & 16 & 8 & 4 & 0 & 1 & 0 & 0 & 0 & 0 & 3 & 40 \\
Control & 0 & 0 & 1 & 0 & 1 & 2 & 1 & 2 & 2 & 0 & 0 & 10 \\
\hline
\end{tabular}


Both $M$. anisopliae s.l. isolates displayed a similar efficacy, although E9 exerted a somewhat faster effect than IBCB 425. This difference in acaricidal activity between the two isolates was nonsignificant because both killed $90 \%$ of the ticks in 4 days and both killed $90 \%$ of the ticks in 11 days. Nonetheless, the acaricidal activity of M. anisopliae s.l. was high when compared to the activity in similar tests against other tick species with the same fungus (Garcia et al. 2004, 2011). Unfortunately, direct comparison with the efficacy of isolates from other studies is hampered by the use of dissimilar fungus stocks, conidial suspension concentrations and tick stages, an issue that will be further scrutinized in the future.

The use of entomopathogenic fungi against ticks has long been pursued, but in vitro efficacy in laboratory tests has not been achieved in corresponding field tests (Garcia et al. 2011). Among other factors, solar UV radiation in the field is harmful to fungi (Braga et al. 2001), and an appropriate formulation in which the conidia are suspended might circumvent such drawbacks (Hedimbi et al. 2008). Considering observations from the results presented herein, if a stable $M$. anisopliae s.l. conidia formulation is achieved, control of ticks on domestic animals, including A. parvum, will be feasible.

Acknowledgements This research was supported by Conselho Nacional de Desenvolvimento Científico e Tecnológico-CNPq (Academic Career Research Fellowship to M.P.J. Szabó) and Fundação de Amparo à Pesquisa do Estado de Minas Gerais-FAPEMIG and Fundação de Apoio ao Desenvolvimento do Ensino, Ciência e Tecnologia do Estado de Mato Grosso do Sul (Fundect, MS)—Governo do Estado de Mato Grosso do Sul.

\section{References}

Basso LMS, Monteiro AC, Belo MAA, Soares VE, Garcia MV, Mochi DA (2005) Controle de larvas de Boophilus microplus por Metarhizium anisopliae em pastagens infestadas artificialmente. Pesq Agr Bras 40:595-600

Bittencourt VREP, Mascarenhas AG, Faccini JLH (1999) The penetration of the fungus Metarhizium anisopliae on Boophilus microplus in experimental conditions. Cien Rural 29:351-354

Bittencourt VREP, Bahiense TC, Fernandes EKK, Souza EJ (2003) Avaliação da ação in vivo de Metarhizium anisopliae (Metschnikoft, 1879) Sorokin, 1883 aplicado sobre Brachiaria decumbens infestada com larvas de Boophilus microplus (Canestrini 1883) (Acari: Ixodidae). Rev Bras Parasitol Vet $12: 38-42$

Braga GUL, Flint SD, Miller CD, Anderson AJ, Roberts DW (2001) Variability in response to UV-B among species and strains of Metarhizium isolated from sites at latitudes from $61^{\circ} \mathrm{N}$ to $54^{\circ} \mathrm{S}$. J Invertebr Pathol 78:98-108

De Meneghi D, Stachurski F, Adakal H (2016) Experiences in tick control by acaricide in the traditional cattle sector in Zambia and Burkina Faso: possible environmental and public health implications. Front Public Health 4:1-11

Francisco EA, Mochi DA, Correia ACB, Monteiro AC (2006) Influence of culture media in viability test of conidia of entomopathogenic fungi. Cienc Rural 36:1309-1312

Garcia MV, Monteiro AC, Szabó MPJ (2004) Colonização e lesão em fêmeas ingurgitadas do carrapato Rhipicephalus sanguineus causadas pelo fungo Metarhizium anisopliae. Cienc Rural 34:1513-1518

Garcia MV, Monteiro AC, Szabó MPJ, Prette N, Bechara GH (2005) Mechanism of infection and colonization of Rhipicephalus sanguineus eggs by Metarhizium anisopliae as revealed by scanning eletron microscopy and histopathology. Braz J Microbiol 36:368-372

Garcia MV, Monteiro AC, Szabó MPJ, Mochi DA, Siumi LD, Carvalho WM, Tsuruta AS, Barbosa JC (2011) Effect of Metarhizium anisopliae fungus on off-host Rhipicephalus (Boophilus) microplus form tick-infested pasture under cattle grazing in Brazil. Vet Parasitol 181:267-273

Hedimbi M, Kaaya GP, Singh S, Chimwamurombe PM, Gindin G, Glazer I, Samish M (2008) Protection of Metarhizium anisopliae conidia from ultra-violet radiation and their pathogenicity to Rhipicephalus evertsi evertsi ticks. Exp Appl Acarol 46:149-156 
Higa LOS, Garcia MV, Barros JC, Koller WW, Andreotti R (2015) Acaricide resistance status of the Rhipicephalus microplus in Brazil: a literature overview. Med Chem 5:326-333

Lado P, Nava S, Labruna MB, Szabo MPJ, Durden LA, Bermudez S, Montagna M, Sanchez AC, Beati L (2016) Amblyomma parvum Aragão, 1908 (Acari: Ixodidae): phylogeography and systematic considerations. Ticks Tick Borne Dis 7:817-827. https://doi.org/10.1016/j.ttbdis.2016.03.017

Lopes RB, Alves SB, Padulla LFL, Pérez CA (2007) Eficiência de formulações de Beauveria bassiana e Metarhizium anisopliae para o controle de ninfas de Amblyomma cajennense (FABRICIUS, 1787). Rev Bras Parasitol Vet 16:27-31

Monteiro SG, Bittencourt VREP, Daemon E, Faccini JLH (1998) Pathogenicity under laboratory conditions of the fungi Beauveria bassiana and Metarhizium anisopliae on larvae of the tick Rhipicephalus sanguineus (Acari: Ixodidae). Rev Bras Parasitol Vet 7:113-116

Nava S, Szabó MPJ, Mangold AJ, Guglielmone AA (2008) Distribution, hosts, 16S rDNA sequences and phylogenetic position of the Neotropical tick Amblyomma parvum (Acari, Ixodidae). Ann Trop Med Parasitol 102:409-425

Nieri-Bastos FA, Lopes MG, Cançado PHD, Rossa GA, Faccini HJL, Gennari SM, Labruna MB (2014) Candidatus Rickettsiae andeanae, a spotted fever group agent infecting Amblyomma parvum ticks in two Brazilian biomes. Mem Inst Oswaldo Cruz 10:1-3

Olegário MMM, Gerardi M, Tsuruta AS, Szabó MPJ (2011) Life cycle of the tick Amblyomma parvum Aragão, 1908 (Acari: Ixodidae) and suitability of domestic hosts under laboratory conditions. Vet Parasitol 179:203-208

Pacheco RC, Echaide IE, Alves RN, Beletti ME, Nava S, Labruna MB (2013) Coxiella burnetii in ticks, Argentina. Emerg Infect Dis 19:344-346

Polar P, De Muro MA, Kairo MTK, Peterkin D, Moore D, Pegram R, John SA (2005) Assessment of fungal isolates for development of a myco-acaricide for cattle tick control. Vector Borne Zoonotic Dis 5:276-284

Ramos VN, Osava CF, Piovezan U, Szabó MPJ (2014) Ticks on humans in the Pantanal wetlands, Brazil. Ticks Tick Borne Dis 5:497-499

Ramos VN, Piovezan U, Franco AHA, Rodrigues VS, Nava S, Szabó MPJ (2016) Nellore cattle (Bos indicus) and ticks within the Brazilian Pantanal: ecological relationships. Exp Appl Acarol 68:227-240. https://doi.org/10.1007/s10493-015-9991-3

Szabó MPJ, Olegário MMM, Santos ALQ (2007) Tick fauna from two locations in the Brazilian savannah. Exp Appl Acarol 43:73-84

Tomassone L, Nuñez P, Gurtler R, Ceballos LA, Orozco MA, Kitron UD, Farber M (2008) Molecular detection of Ehrlichia chaffeensis in Amblyomma parvum ticks, Argentina. Emerg Infect Dis 14:1953-1955 